\title{
ARTICLE Methylphenidate's effects on thalamic metabolism and functional connectivity in cannabis abusers and healthy controls
}

\author{
Șükrü Barıș Demiral (iD ${ }^{1,2}$, Dardo Tomasi ${ }^{1}$, Corinde E. Wiers ${ }^{1}$, Peter Manza ${ }^{1}$, Ehsan Shokri-Kojori (iD ${ }^{1}$, Yana Studentsova ${ }^{1}$, \\ Gene-Jack Wang ${ }^{1}$ and Nora D. Volkow ${ }^{1,3}$
}

Methylphenidate (MPH) is a first line treatment for ADHD and is also misused as a purported cognitive enhancer, yet its effects on brain function are still poorly understood. Recent functional magnetic resonance imaging (fMRI) studies showed that MPH altered cortico-striatal resting functional connectivity (RFC). Here we investigated the effects of MPH in thalamic connectivity since the thalamus modulates striato-cortical signaling. We hypothesized that MPH would increase thalamic connectivity and metabolism, and that this response would be blunted in cannabis abusers. For this purpose, we measured RFC in seven thalamic nuclei using fMRI and brain glucose metabolism using positron emission tomography (PET) and ${ }^{18}$ F-fluorodeoxyglucose (FDG) in sixteen healthy controls and thirteen participants with cannabis use disorder (CUD) twice after placebo and after MPH $(0.5 \mathrm{mg} / \mathrm{kg}$, iv). MPH significantly increased thalamo-cerebellar connectivity and cerebellar metabolism to the same extent in both groups. Group comparisons revealed that in CUD compared to controls, metabolism in nucleus accumbens was lower for the placebo and MPH measures, that MPH-induced increases in thalamic metabolism were blunted, and that enhanced negative connectivity between thalamus and accumbens in CUD was normalized by MPH (reducing negative connectivity). Our findings identify the thalamus as a target of MPH, which increased its metabolism and connectivity. The reduced metabolism in nucleus accumbens and the disrupted thalamo-accumbens connectivity (enhanced negative connectivity) in CUD is consistent with impaired reactivity of the brain reward's circuit. MPH's normalization of thalamo-accumbens connectivity (reduced negative connectivity) brings forth its potential therapeutic value in CUD, which merits investigation.

Neuropsychopharmacology (2019) 44:1389-1397; https://doi.org/10.1038/s41386-018-0287-2

\section{INTRODUCTION}

Methylphenidate (MPH) is a psychostimulant medication widely used for therapeutic purposes, but is also globally misused both for its purportedly cognitive enhancing properties [1]. It is accepted that its pharmacological effects are mediated by its dopaminergic and noradrenergic enhancing effects $[2,3]$, which result from its blockade of dopamine (DA) and norepinephrine (NE) transporters $[4,5]$.

Positron emission tomography (PET) studies have confirmed that MPH's effects are strongly tied to tonic levels of DA release [6]. MPH might also increase presynaptic striatal DA synthesis as suggested by findings from a PET study using $\left[{ }^{18} \mathrm{~F}\right]$ fluorodopamine ([ $\left.\left.{ }^{18} \mathrm{~F}\right] \mathrm{DOPA}\right)$ [7]. Additionally functional brain imaging studies have shown that MPH increases brain glucose metabolism (measured with PET and ${ }^{18} \mathrm{~F}$-fluorodeoxyglucose (FDG)) in a diverse set of brain regions including cingulate gyrus, thalamus, and cerebellum during non-stimulation conditions [8-10]. However when given to healthy controls while performing a cognitive (numerical calculation) task, MPH attenuated the task-induced increases in regional brain glucose metabolism, consistent with an enhancement of neuronal efficacy [11]. MPH's effects on brain glucose metabolism are also influenced by prior drug exposure histories. Specifically, in participants with a cannabis use disorder (CUD) the effects of MPH in regional brain glucose metabolism were markedly attenuated [10]; whereas they were enhanced in participants with an alcohol use disorder [8]. Additionally, MPH's behavioral effects in healthy controls [12] and in participants with substance use disorders is state dependent (i.e. influenced by the state of arousal, exposure to cues, task difficulty) [13].

The effects of MPH on brain function have also been assessed with functional magnetic resonance imaging (fMRI) using taskbased and resting-state measures in healthy controls [14], and in CUD participants [15]. In healthy controls, MPH reduced BOLD responses to gain and loss trials and to prediction errors [14]; reduced resting functional connectivity (RFC) between anterior cingulate cortex and thalamus when the whole thalamus was selected as seed [16], and reduced RFC between globus pallidus, ventral pallidum, cerebellum and the nucleus accumbens (NAC) [17], which is a ventral striatal region underlying motivation and reward [18].

Though MPH's effects on striato-cortical connectivity are likely to mediate some of its therapeutic and rewarding effects [3], its effects on thalamic activity and connectivity are also likely to be relevant. The thalamus is a relay between midbrain dopamine

\footnotetext{
${ }^{1}$ National Institute on Alcohol Abuse and Alcoholism, National Institutes of Health, Bethesda, MD, USA; ${ }^{2}$ Behavioral Biology Branch, Center for Military Psychiatry and Neuroscience, Walter Reed Army Institute of Research, Silver Spring, MD, USA and ${ }^{3}$ National Institute on Drug Abuse, National Institutes of Health, Bethesda, MD, USA Correspondence: Şükrü Barış. Demiral (sukru.demiral@nih.gov) or Nora D. Volkow (nvolkow@nida.nih.gov)
} 
projections into the striatum and the cortex and it's a main target of noradrenergic projections from the locus coeruleus [19], positioning it as a critical node in the networks that mediate MPH's rewarding and therapeutic effects. As it relates to reward, the NAc connects into the thalamus (via its projections to the ventral pallidum), where its primary output is the mediodorsal thalamic nucleus (MD) [20]. Preclinical studies with cocaine, a drug pharmacologically similar to MPH [5] also implicate the posterior paraventricular thalamic nucleus (PVT), which sends glutamatergic projections to NAc and contains D2-like dopamine receptors [21], in its rewarding effects [22]. On the other hand the dorsal lateral geniculate thalamic nucleus has been implicated in MPH's performance enhancement to a visual detection task, presumably via its noradrenergic modulation [23].

Using graph theoretical approaches [24], MPH was shown to decrease short-range connectivity in putamen and thalamus in controls and cocaine abusers [25]. However, the comparison of MPH's effects on long-range thalamic connectivity between healthy controls and drug-abusers has been minimally investigated. In particular we were interested on assessing its effects in CUD since the thalamus contains high levels of cannabinoid CB1 receptors, which are implicated in its oscillatory activity and in arousal [26]. Moreover, in CUD participants, the thalamus was shown to be involved in reactivity to cannabis cues [27]; to have enhanced local connectivity [28] and to have increased levels of a $4 \beta 2$ nicotinic receptors [29]. The thalamus is structurally and functionally heterogeneous including distinct connectivity patterns [30-32], and metabolic activity for the various thalamic nuclei [33]; and thus in our study we investigated the effects of MPH across thalamic sub-segments and compared its effects in healthy controls to that in CUD participants.

For this purpose we measured brain glucose metabolism, which is a marker of brain activity $[34,35]$ using PET and FDG, as well as long-range RFC via fMRI seed-based analysis using seven thalamic subregions, referred here as thalamic seeds [31] in healthy controls (HC; $n=16,8$ females) and in participants with CUD (CUD; $n=13,7$ females). Each of these thalamic seeds dominantly project to motor, sensory, occipital, frontal, premotor, parietal, temporal cortical regions via white matter tracks (Sup. Fig 1, shows the location of the thalamic seeds referred to as TH1 to TH7).

We hypothesized that MPH would increase thalamic metabolism and long-range RFC and that the changes in thalamic metabolism would be associated with changes in thalamic connectivity. We also hypothesized that MPH's effects on thalamic metabolism and connectivity would be blunted in CUD compared to HCs in whom we previously reported reduced behavioral and brain reactivity to $\mathrm{MPH}[36,37]$. All participants underwent scanning on two separate sessions, after MPH (0.5 mg/kg iv), and after Placebo (saline iv).

\section{MATERIALS AND METHODS}

Participants

Thirteen CUD (7 females; age $27.2 \pm 7.6$ ) and sixteen HC (8 females; age $28.2 \pm 4.9$ ) completed the study (Table 1). Participants were recruited from advertisements in local newspapers, and signed a written informed consent approved by the Committee on Research in Human Subjects at Stony Brook University (IRB net number 225114). See Supplementary Methods for the details of participant selection criteria.

Study design and drug administration

The study was a single blind design: i.e., subjects were blind to the drugs (MPH or Placebo; $\mathrm{PL}$ ) received. There were two scan days; one for PL administration and one for MPH administration. In each day, either $\mathrm{MPH}(0.5 \mathrm{mg} / \mathrm{kg})$ or PL (3 cc saline) were injected intravenously $90 \mathrm{~min}$ prior to the PET-FDG scan procedure, which was followed by the fMRI scanning session (150-180 min after MPH or PL injection). See Supplementary Methods for the details of PET and $\mathrm{FMRI}$ data acquisition.

Seed-based fMRI connectivity analysis

Standard seed-voxel correlation analyses were used to estimate the functional connectivity of the thalamic partitions in the Behrens atlas [31] (Sup. Fig. 1). Pearson correlations were used to compute the strength of the functional connectivity between the average time-varying signals within each of the thalamic partitions (i.e. seeds, bilateral) and the rest of the brain voxels. See Supplementary Methods for the details of RFC analysis.

Thalamic seed metabolic analyses

Average absolute metabolic values were extracted for whole thalamus and each of the seven thalamic seeds (bilateral) for each participant and condition from the PET-FDG images and were used to compare GROUP, DRUG, and SEED effects.

Metabolic changes and correlations across thalamic seeds and target ROIs

After finding resting state seed-based connectivity clusters as defined above, brain metabolism was further evaluated with region-of-interest $(\mathrm{ROI})$ analysis to obtain signal values in the selected target volumes (Supplementary Methods).

We focused on the ROls showing significant drug effects in RFC $(\mathrm{MPH}>\mathrm{PL}$ or $\mathrm{MPH}<\mathrm{PL})$. We first calculated and compared changes in glucose metabolism in these ROls. Then, in the final step, metabolic changes in these ROIs were compared to the metabolic changes in the thalamic seeds -which project onto those ROls- via Pearson correlation ( $r$ ) analyses; to assess whether metabolic changes in thalamic seeds correlated with the metabolic changes in these target brain regions.

\section{Statistical approach}

For voxel-wise analysis of RFC data to assess thalamic connectivity we used a flexible factorial general lineal model with DRUG (within-subject) and GROUP (between-subject) $(2 \times 2)$ factors in SPM12. Follow-up post-hoc analyses were conducted with paired (for DRUG contrasts; MPH $>\mathrm{PL}$, and $\mathrm{PL}>\mathrm{MPH}$ ) or unpaired (for GROUP contrasts; HC > CUD, and CUD > HC) F-tests in SPM12. We first used a standard double threshold method; first chose a cluster forming voxel threshold of $p<0.005$ with $k>50$ (minimum of 50 neighboring voxels), and then applied a threshold of $p<0.05$ to correct for family-wise error (FWE) across the $p$-values of the surviving clusters as described elsewhere [38]. Following this standard SPM approach, we additionally used Bonferroni to correct for multiple observations planned across thalamic seeds (i.e., for seven thalamic seeds cluster FWER p-threshold was set to $0.05 / 7$ (0.0072), see Table 2). The surviving clusters were then used to form ROls around the voxel with peak intensity in that cluster for further comparisons.

For the brain metabolic images (PET-FDG) we performed a 3way ANOVA using GROUP, DRUG and SEED $(2 \times 2 \times 7)$ to assess the effects of MPH across the thalamic seeds.

For the metabolic analysis of the target ROls, we first picked ROIs showing significant effect of RFC for DRUG (MPH $>P L$, shaded rows in Table 2; note that there were no significant RFC effects for the contrast PL $>M P H$ that survived correction for multiple comparisons). We then ran a 2-way ANOVA using GROUP and DRUG $(2 \times 2)$ for the metabolic changes in each of these regions. Finally, we measured the metabolic changes (MPH-PL) in the thalamic seeds as well as their target ROls to assess the correlation between MPH-induced metabolic changes in these connected regions using pearson product moment correlation analyses. The $p$-values were adjusted for multiple comparisons via Bonferroni correction on all of these analyses. ANOVA and correlation analyses on the extracted values from the thalamic 
Table 1. Participant demographics

\begin{tabular}{|c|c|c|c|c|c|c|c|}
\hline & \multicolumn{2}{|l|}{ CUD } & \multicolumn{2}{|l|}{$\mathrm{HC}$} & \multicolumn{3}{|c|}{$p$-values } \\
\hline Age, years & $23.23(2.33)$ & $31.78(9.64)$ & $27.63(5.65)$ & $28.78(4.49)$ & NS & 0.052 & NS \\
\hline Years of education & $12.83(1.33)$ & $12.42(1.52)$ & $13.21(1.64)$ & $12.98(1.14)$ & NS & NS & NS \\
\hline $\mathrm{BMI}$ & $22(3.28)$ & $26.84(2.58)$ & $24.12(2.05)$ & $24.37(2.86)$ & NS & 0.032 & 0.033 \\
\hline Cigarette smokers & 2 active & 2 active & 1 active & 1 active & NS & NS & NS \\
\hline Days/week & $6.86(0.38)$ & $6.5(0.84)$ & - & - & - & NS & - \\
\hline Joints/day & $5.43(3.6)$ & $4.5(3.27)$ & - & - & - & NS & - \\
\hline Years abuse & $7(3.74)$ & $14.33(10.76)$ & - & - & - & NS & - \\
\hline MDQ Sum & $4.86(2.54)$ & $4.83(2.14)$ & - & - & - & NS & - \\
\hline MPQ PEM & $41.43(12.07)$ & $51.67(13.03)$ & $54(4.93)$ & $52.88(3.76)$ & 0.038 & NS & NS \\
\hline
\end{tabular}

seeds and target ROls were conducted using $\mathrm{R}$ software version 3.4.3 [39].

\section{RESULTS}

Head motion

The average frame displacement (FD) did not differ between HC $(0.228 \pm 0.144 \mathrm{~mm}$ and $0.208 \pm 0.076 \mathrm{~mm}$ for $\mathrm{PL}$ and $\mathrm{MPH})$, and CUD $(0.225 \pm 0.089 \mathrm{~mm}$ and $0.185 \pm 0.053 \mathrm{~mm}$ for $\mathrm{PL}$ and $\mathrm{MPH})$. There was no effect of drug on FD nor was there an interaction between drug $\times$ group $(F<1)$.

Thalamic seed functional connectivity

We examined long-range connectivity of the thalamus using seven bilateral seed regions that project to motor, sensory, occipital, frontal, premotor, parietal, temporal cortical regions via white matter tracks (referred to as $\mathrm{TH} 1$ to $\mathrm{TH} 7$, respectively).

We found that only five of these seeds, namely motor (TH1), sensory (TH2), premotor (TH5), parietal (TH6), and temporal (TH7) seeds formed surviving resting state connectivity clusters, resulting in a total of nine thalamo-cortical or thalamo-cerebellar connectivity pairs throughout the brain. This was because three seeds, namely premotor, sensory, and motor seeds, formed more than one surviving connectivity cluster, while two of the thalamic seeds, namely parietal and temporal seeds, formed only one surviving connectivity cluster. The occipital (TH3) and frontal (TH4) seeds did not form any surviving connectivity cluster (Table 2). Thus, five of the seven seeds are reported in the paper.

Drug effects. Thalamic seed 1 (motor) showed increased RFC with right cerebellum for MPH than for PL $(p<0.001)$ (Fig. 1a). Thalamic seed 2 (sensory) showed increased RFC with right cerebellum for MPH than for PL $(p<0.006)$ (Fig. 1b).

Group effects. Thalamic seed 1 (motor) showed stronger RFC with left pars triangularis $(p<0.006)$ and left supramarginal gyrus $(p<$ 0.001 ) (Fig. 2a, c), and weaker RFC with visual areas for CUD than $\mathrm{HC}(p<0.005)$ (Fig. 2e). Thalamic seed 2 (sensory) showed stronger RFC with right parietal cortex, for CUD than HC $(p<0.005)$ (Fig. 2b). Thalamic seed 6 (parietal) showed stronger RFC with left parietal cortex, for HC than CUD $(p<0.005)$ (Fig. 2f). Thalamic seed 7 (temporal) showed stronger RFC with right temporo-parietal cortex for CUD than HC $(p<0.001)$ (Fig. 2d).
Group $\times$ drug interaction. Thalamic seed 5 (premotor) showed a GROUP and a DRUG $\times$ GROUP interaction effect with nucleus accumbens (NAc) cluster (left hemisphere dominant, henceforth named as left NAc) (Fig. 1c). The CUD group showed negative RFC with left NAc for PL that was significantly larger than for $\mathrm{HC}(p<$ $0.005)$. The DRUG $\times$ GROUP effect $(p<0.005)$ (Fig. 1c) was due to MPH's reducing negative RFC in CUD $(p<0.05)$ whereas it increased negative RFC in $\mathrm{HC}(p<0.01)$.

Thalamic metabolism: effects of GROUP and MPH

Absolute metabolic values (mean and SD; expressed as micromol/ $100 \mathrm{~g} / \mathrm{min}$ ) for the whole thalamus in HC and CUD for placebo and $\mathrm{MPH}$ were as follows: for HC: PL condition, 26.66 (6.11), MPH condition, 29.56 (7.05); for CUD: PL condition, 26.76 (4.87), MPH condition, 27.35 (4.90). We computed the mean metabolic value per thalamic seed, per subject per condition, and ran a 3-way ANOVAs $(2 \times 2 \times 7$; GROUP $\times$ DRUG $\times$ SEED). Comparisons showed a trend effect of GROUP, where HC showed higher values than CUD, $F(1,28)=3.77, p=0.052$. MPH significantly increased thalamic metabolism compared to $\mathrm{PL}, \mathrm{F}(1,28)=11.88, p<0.001$. There was also a significant DRUG and GROUP interaction effect, $\mathrm{F}(1,28)=4.54, p<0.05$; driven by a significant increase in thalamic metabolism with MPH in $\mathrm{HC}, \mathrm{F}(1,28)=10.87, p<0.01$, but not in CUD $(F<1)$. There was also a main effect of SEED, $F(6,34)=14.67$, $p<001$, where some thalamic seeds (i.e., TH4, projecting to frontal regions) had highest baseline metabolism compared to the others (Sup. Fig. 2).

Metabolic changes on target ROls due to GROUP and MPH administration

We contrasted the metabolic changes in the three target ROls that were found to be significantly affected by $\mathrm{MPH}$ in the RFC analyses, namely (i) right cerebellum ROI connected to motor seed (TH1), (ii) right cerebellum ROI connected to sensory seed (TH2) (these two ROls showed a main effect of MPH on RFC), and (iii) NAc ROI connected to pre-motor seed (TH5) (which showed an interaction between GROUP and DRUG in RFC). For this purpose we compared the metabolic values with ANOVA to assess GROUP and DRUG effects and their interactions in these ROls (Fig. 3).

Right cerebellum ROls. MPH increased glucose metabolism in the right cerebellar ROIs compared to $\mathrm{PL}$ both for the ROI formed by the motor seed $(F(1,28)=22.64$, Fig. 3ai) and the ROI formed by 
MPH related connectivity changes between thalamic seeds and their right cerebellum and left NAc ROIs
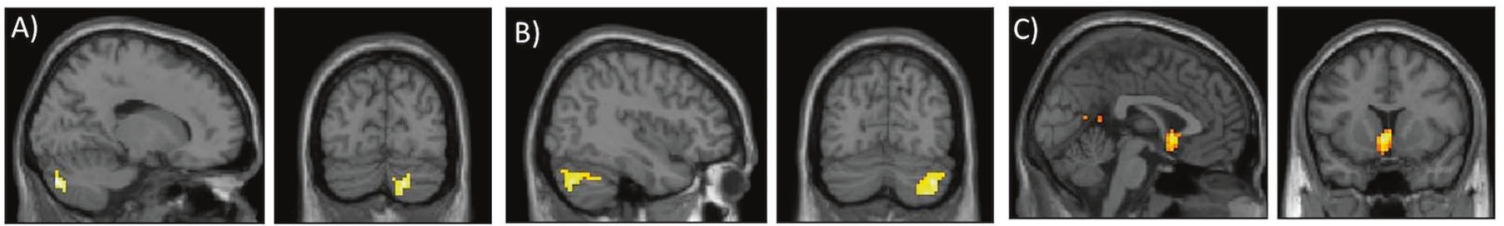

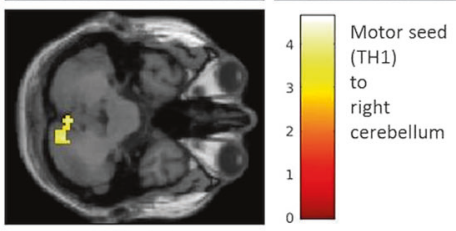

$\mathrm{MPH}>\mathrm{PL}$

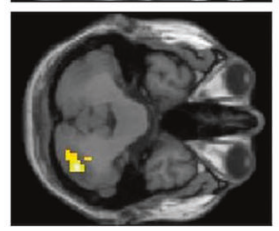

$\mathrm{MPH}>\mathrm{PL}$

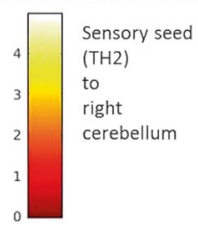

CUD: $\mathrm{MPH}>\mathrm{PL}, \mathrm{MPH}$ decreases negative connectivity

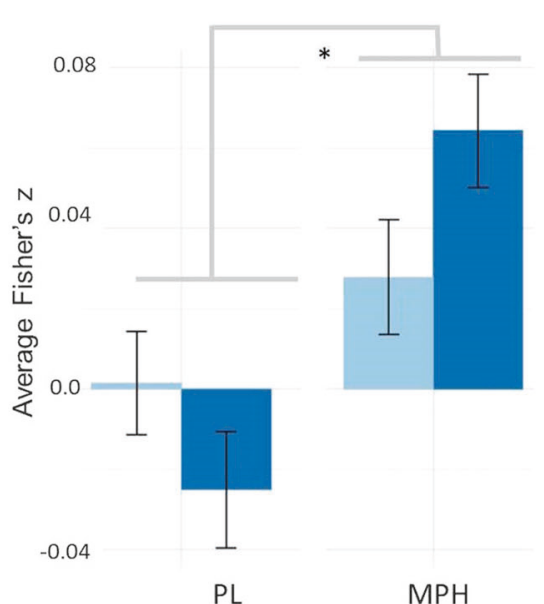

A) Motor seed (TH1) to R. cerebellum ROI

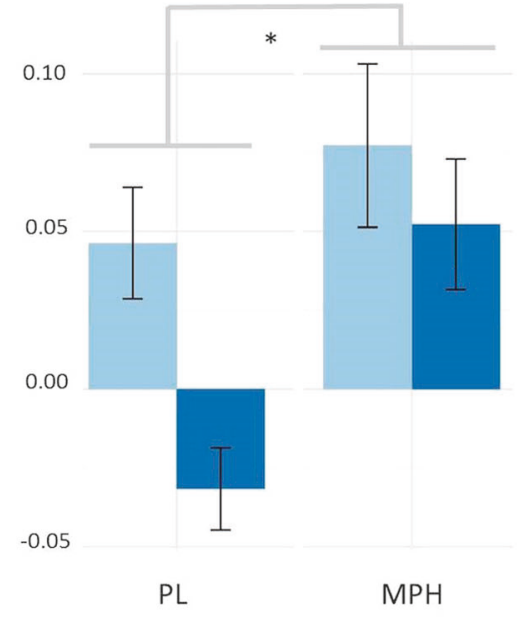

B) Sensory seed ( $\mathrm{TH} 2)$ to R. cerebellum ROI

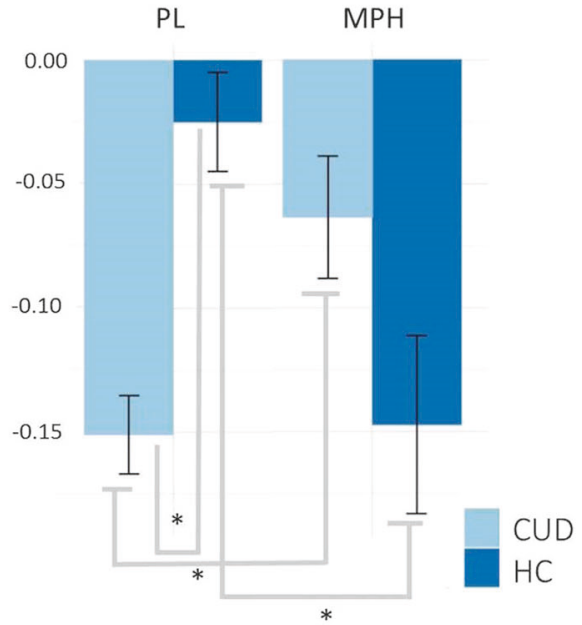

C) Pre-motor seed (TH5) to L. NAc ROI

Fig. $1 \mathrm{MPH}$-induced changes in thalamic connectivity in cerebellum and left NAc. Top row: brain regions with strong seed-based connectivity modulation with a motor, $\mathbf{b}$ sensory, and $\mathbf{c}$ pre-motor thalamic seeds are shown. Thalamic seed names are indicated near the color scale along with their target clusters shown in the figures. In $\mathbf{a}$ and $\mathbf{b}$, the scale shows $t$-values contrasting MPH $>$ PL. In $\mathbf{c}$, scale shows F-value (contrasting interaction between DRUG and GROUP). MPH impacts connectivity on thalamo-cerebellar networks in both groups, that was strongest for the right cerebellum with the sensory and motor thalamic seeds. MPH's effects on pre-motor thalamic seed connectivity with NAc differed between groups; in CUD it decreased negative connectivity whereas in HC it increased negative connectivity. Initial clustering threshold was chosen as $p=0.005$, with $k>50$; final pFWE $<0.0072$. Bottom row: drug effects on connectivity across subjects are shown in barplots. Effect of $\mathrm{MPH}$ as compared to PL in HC and CUD in resting connectivity ROls formed by motor, sensory and pre-motor thalamic seeds. Mean and SD of $\mathrm{z}$-scores of the correlations are plotted across subjects. a Motor thalamic seed connectivity with right cerebellum was increased by MPH. b Sensory thalamic seed connectivity with right cerebellum was increased by MPH. c Pre-motor thalamic seed connectivity with NAc showed that for PL CUD showed greater negative connectivity than for $\mathrm{HC}$ and MPH reduced the negative connectivity in CUD whereas in HC it had the opposite effect enhancing it

the sensory seed $(F(1,28)=9.55, p<0.01)$, Fig. 3aii), consistent with the increases in RFC.

Left NAc ROI. In the NAc ROI, MPH did not significantly affect metabolism, instead $\mathrm{HC}$ showed higher glucose metabolism than CUD $(F(1,28)=31.74, p<0.001$; Fig. 3aiii) both for the placebo and the MPH conditions.

Correlations between metabolic changes (MPH-PL) in thalamic seeds and their target ROls

Since MPH affected metabolism on both target ROls and the thalamic seeds, we calculated the correlations between the metabolic changes induced by MPH (MPH-PL) between each of the three thalamic seeds and their corresponding ROls across subjects (i.e., motor, sensory and pre-motor thalamic seeds and their corresponding target ROls, Fig. 3b).
Right cerebellum ROls. The metabolic changes in the motor thalamic seed and its corresponding right cerebellar target ROI were correlated across all participants $(r=0.69, p<0.001)$ and separate analyses by group showed they were correlated in $\mathrm{HC}$ $(r=0.803, p<0.001)$, but not in CUD $(r=0.470, p>0.1)$ (Fig. 3bi). Similarly the correlations between metabolic changes in the sensory thalamic seed region and its right cerebellar target ROI were significant across all participants $(r=0.709, p<0.001)$, and separate analyses by group showed they were significant in $\mathrm{HC}$ $(r=0.872, p<0.001)$, but not in CUD $(r=0.457, p>0.1)$ (Fig. 3bii).

Left NAc ROI. The correlations between metabolic changes in the pre-motor thalamic seed and its left NAc target ROI were significant across all participants $(r=0.671, p<0.001)$ and separate analyses by group showed they were significant both in HC $(r=0.695, p<0.01)$ and CUD $(r=0.713, p<0.01)$ (Fig. 3biii). 


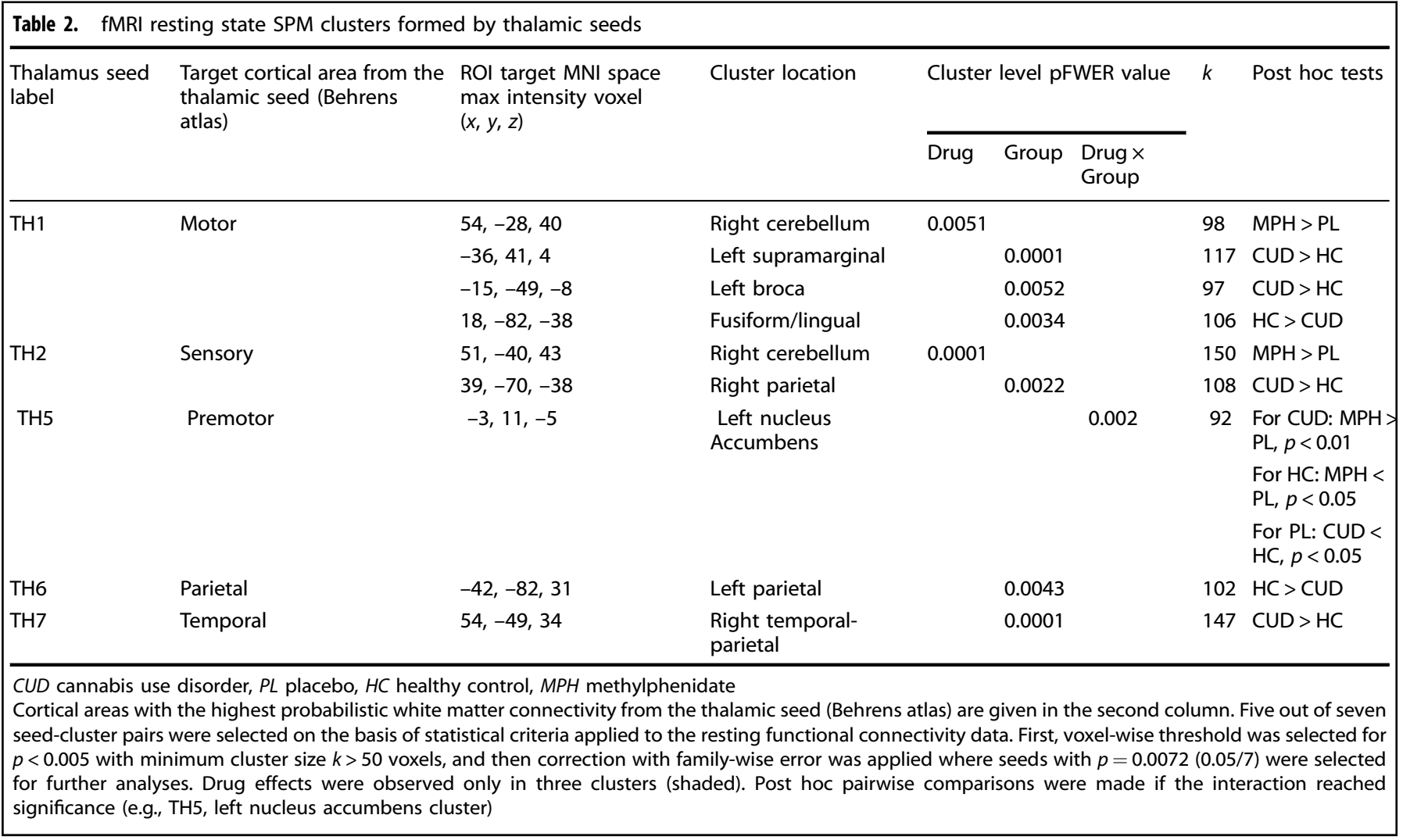

\section{DISCUSSION}

Here, we examined the effects of MPH in seed-based connectivity and brain glucose metabolism in HC and CUD. MPH modulated thalamic functional connectivity in a set of brain regions including NAc. Independent of MPH, thalamic connectivity with NAc and various cortical regions differed between HC and CUD, presumably due to the effects of long-term CUD on brain function or due to pre-existing differences that might have increased vulnerability for CUD. On the other hand, MPH impacted connectivity in interesting ways; for CUD MPH normalized the negative connectivity between NAc and thalamus by bringing connectivity close to the pattern recorded in $\mathrm{HC}$ for the PL condition, whereas in $\mathrm{HC}, \mathrm{MPH}$ increased negative connectivity bringing connectivity close to the pattern recorded in CUD for the PL condition. In addition, MPH increased thalamo-cerebellar connectivity for both $\mathrm{HC}$ and CUD.

While MPH significantly increased metabolism in the cerebellar target ROls, concomitant changes in metabolism between thalamic seeds and cerebellar ROls were significantly correlated only in $\mathrm{HC}$, and not in CUD. The group differences in the correlations was likely driven by the group differences in metabolic effects of MPH on the thalamus, where metabolism was increased in HC-but minimally in CUD. In contrast, in the NAc, a brain region that plays a critical role in drug reward and addiction [18], MPH-induced metabolic changes were correlated with the metabolic changes in the thalamic seed for both in $\mathrm{HC}$ and CUD even though the main effect of MPH on NAc metabolism was not significant, highlighting the inter-subject variability for $\mathrm{MPH}$ effects in NAc metabolism.

\section{RFC changes}

MPH effects. MPH increased connectivity between sensory and motor thalamic seeds and right cerebellum (for both HC and CUD). On the other hand, MPH produced a contrasting connectivity pattern between premotor thalamic seed region and NAc, where MPH effects differed between groups. Specifically, MPH decreased the negative connectivity between pre-motor thalamic seed region and NAC in CUD, while it increased negative connectivity in HC. This finding is in line with previous reports on connectivity changes in NAc [17] and of abnormal dopaminergic responses to MPH challenge in CUD versus controls [10, 40]. The disrupted thalamo-accumbens connectivity in CUD adds to the growing evidence of the importance of thalamic inputs to NAc in drug abuse and addiction [41].

Group effects. We observed group differences in the functional connectivity of a diverse set of regions connecting to thalamic seeds. CUD showed a main effect of hyperconnectivity in motor, sensory and temporal thalamic seeds with many cortical clusters across the brain, including Broca's region and parietal and temporo-parietal regions. Given the widespread distribution of cannabinoid receptors in brain [42], and the complex nature of the interactions between cannabis and other types of drugs with which it is frequently consumed [43], it is not unexpected to find effects of chronic cannabis use on long-range thalamic connectivity. Our findings are consistent with prior imaging studies that identified increases in functional connectivity in subcortical regions (short-range connectivity) in CUD compared to HC [28].

\section{Metabolic changes in the thalamus}

Recent findings showed that glucose metabolism differed across thalamic nuclei, which was tied to cell density differences; for instance medial dorsal regions of thalamus projecting dominantly to prefrontal and temporal regions show higher glucose metabolism, and also higher cell density [33]. Confirming this finding, we showed that thalamic seeds projecting heavily to the frontal cortical regions showed the highest metabolic values. We also showed that MPH significantly increased thalamic glucose 

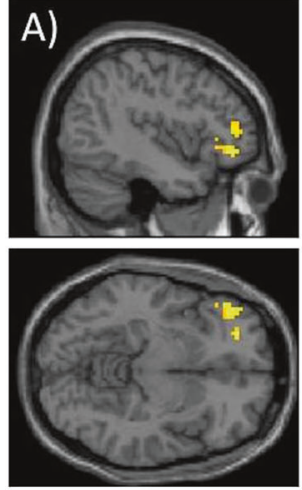

$\mathrm{CUD}>\mathrm{HC}$
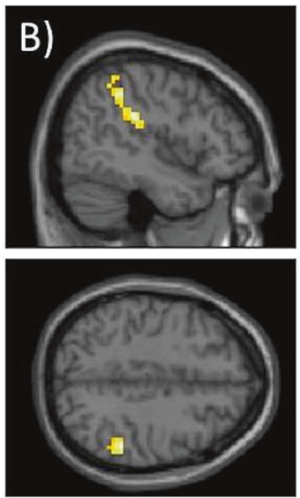

CUD $>\mathrm{HC}$
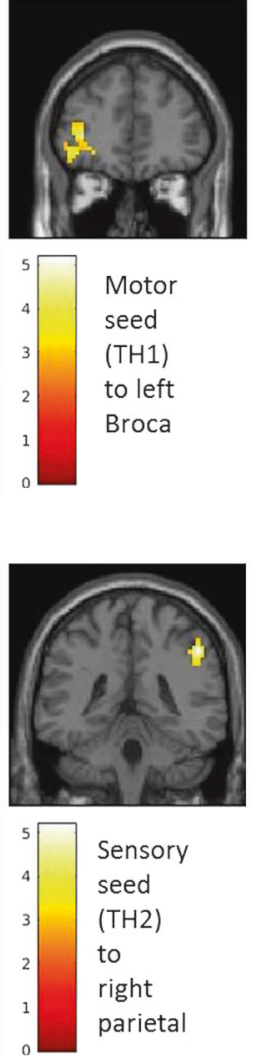

arietal
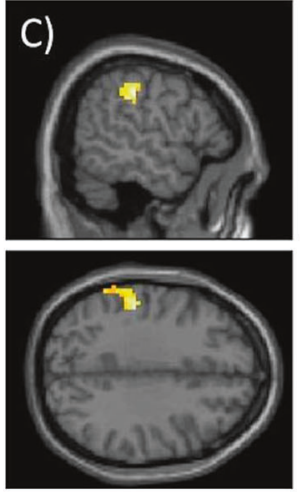

CUD $>\mathrm{HC}$
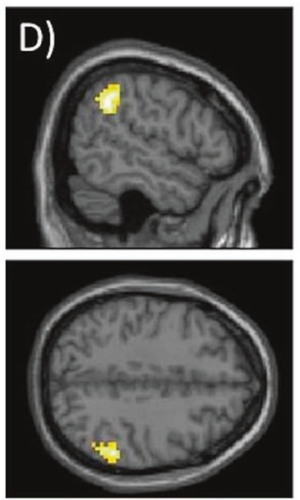

$\mathrm{CUD}>\mathrm{HC}$
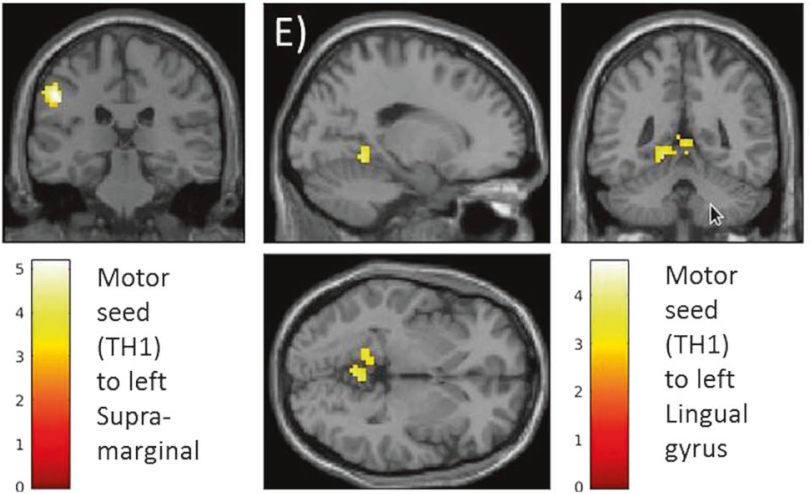

Motor

seed

(TH1)

to left

Lingual

gyrus

HC>CUD
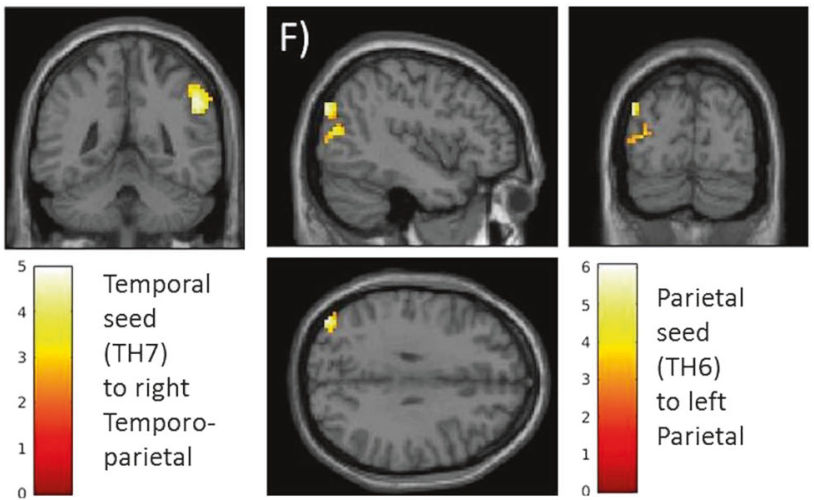

HC $>$ CUD

Fig. 2 Group differences in thalamo-cortical connectivity. Brain regions with significant seed-based connectivity differences between groups. Thalamic seed region names are indicated near the color scale along with their target clusters. In a-d, CUD > HC, for e, and f, HC > CUD. In $\mathbf{a}, \mathbf{c}$ and $\mathbf{e}$, the motor thalamic seed (TH1) showed group differences in connectivity with left Broca, left supramarginal gyrus, and left lingual regions respectively; in $\mathbf{b}$, the sensory seed (TH2) shows group differences in the right parietal region; in d, the temporal seed (TH7) shows group differences in the temporal-parietal region; in f, the parietal seed (TH6) showed group differences in the left parietal region. All tests are $t$-tests with either HC $>$ CUD or CUD $>$ HC contrasts. Initial clustering threshold was chosen as $p=0.005$, with $k>50$; final pFWE $<0.0072$.

metabolism across all seed regions and this effect was mainly driven by HC since CUD had a blunted metabolic response. However we did not observe differences in sensitivity to MPH among sub-sections of the thalamus, revealing the broad effect of MPH over thalamus.

Metabolic changes in target ROls

Cerebellar ROls. The right cerebellar regions showed increases in glucose metabolism (in parallel to increases in RFC) with $\mathrm{MPH}$ and these metabolic changes did not differ between HC and CUD. We recently reported that MPH increased metabolism in whole brain and in whole cerebellar gray matter predominantly in $\mathrm{HC}$, and that $\mathrm{MPH}$-induced metabolic changes were blunted in CUD in a larger sample that included the participants from the current study [10]. The absence of Group and Drug interaction effects on the cerebellar metabolic findings in the current study could be due to the pre-selection of ROIs (determined by RFC analysis), which confined them into smaller cerebellar subregions. Regional specificity of the cerebellum has been documented for diverse cognitive and emotional functions [44]; for sleep [45] and in neurodegenerative diseases [46].

NAC ROI. In NAc we showed a significant Group effect in metabolism, HC had higher metabolism than CUD both in PL and $\mathrm{MPH}$ conditions, which might reflect the deleterious effects of chronic exposure to cannabis in the NAC or that pre-existing lower activity and reactivity of NAc might constitute a risk factor for cannabis consumption.

Concomitant metabolic changes (MPH-PL) in the thalamic seeds and their target ROls

We also observed that MPH-induced metabolic changes in the motor and sensory thalamic seeds were associated with the cerebellar metabolic changes. However these associations were driven by the HC, for they were not significant in CUD. On the other hand, while metabolic changes due to MPH in NAc were not significant due to potentially larger individual variability in $\mathrm{MPH}$ effects, they were significantly correlated with the metabolic changes in the premotor thalamic seed in both HC and CUD. The significant correlation between the changes in metabolism even when the main effect of MPH was non-significant was most likely due to the inter-subject variability for MPH effects in NAC metabolism (i.e., MPH increased metabolism in some participants and decreased it in others) both for HC and CUD, though overall NAC metabolism was significantly higher for HC than for CUD.

Catecholaminergic system: dopaminergic versus noradrenergic The distinct RFC patterns triggered by MPH in cerebellum versus those in NAc could reflect the fact that these brain regions are differentially modulated by noradrenaline and dopamine, both of which are enhanced by MPH. The cerebellum is predominantly influenced by noradrenergic signals from the locus coeruleus, which also extends afferent projections to the thalamus [47]. MPH 


\section{A. Absolute FDG values at the drug-modulated target ROls}

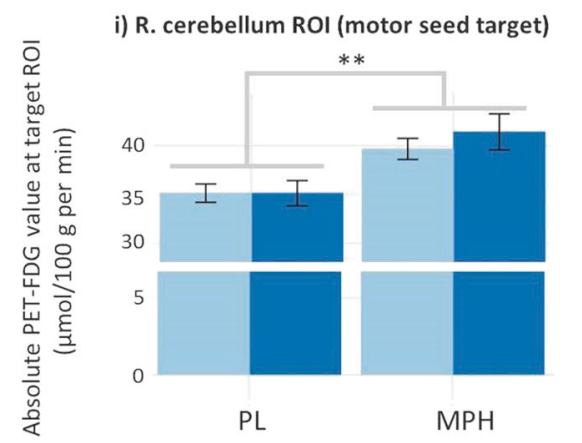

ii) R. cerebellum ROI (sensory seed target)

iii) L. NAc ROI (pre-motor seed target)
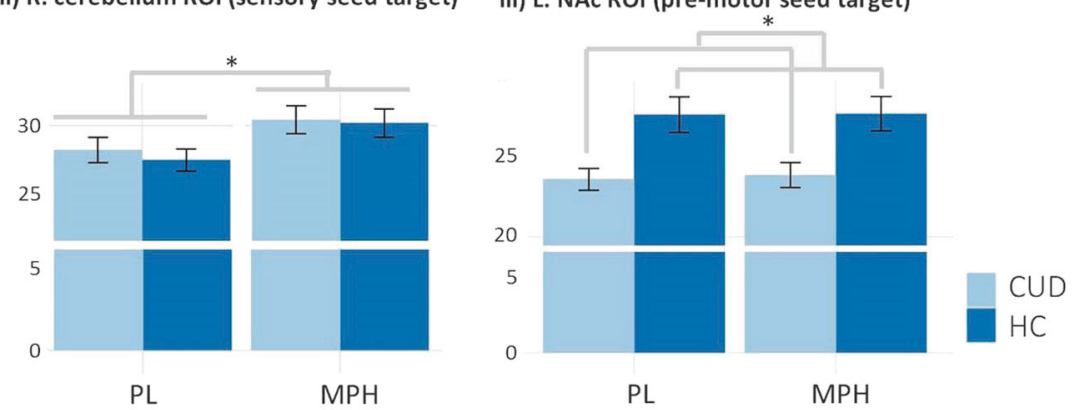

B. Correlations between absolute FDG changes at the drug-modulated target ROIs and their seeds
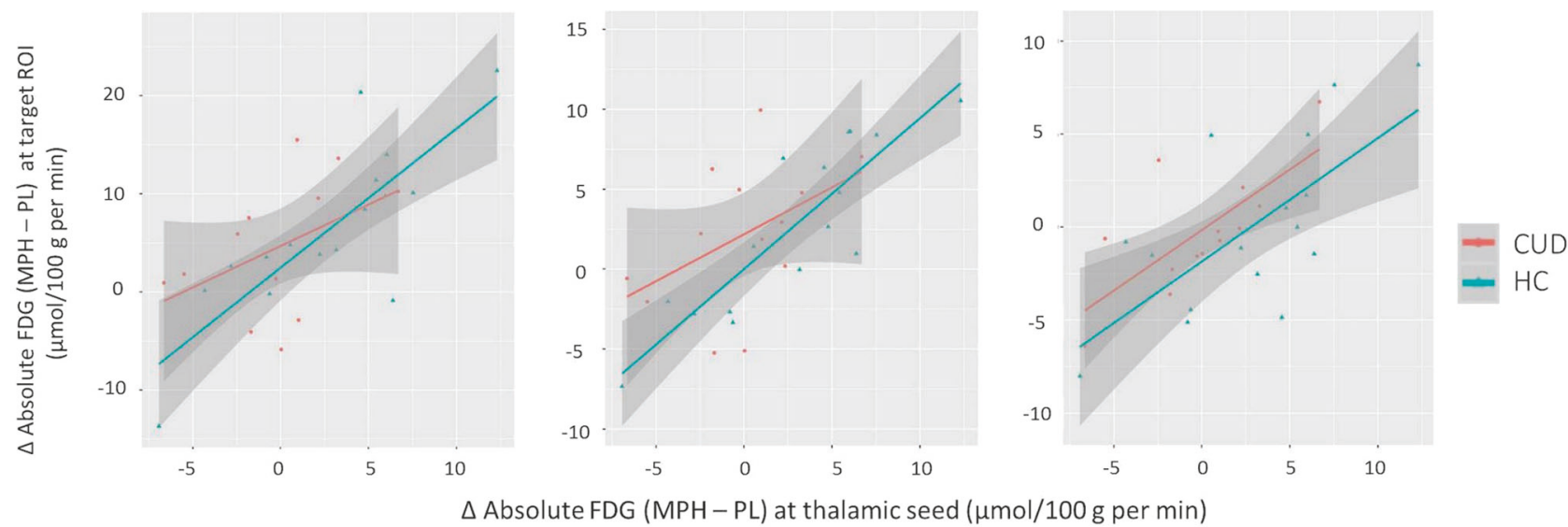

Fig. 3 MPH-induced metabolic changes at target ROls and their corresponding correlations with thalamic seeds. Top row: absolute FDG-PET metabolic measures for PL and MPH on three target ROIs; (i) right cerebellum ROI formed by motor seed, (ii) right cerebellum ROI formed by sensory seed, and (iii) NAc ROI formed by pre-motor seed. Metabolism in the right cerebellum ROls were significantly increased by MPH, independent of GROUP. Metabolism in NAc showed a main effect of GROUP (HC > CUD) but not an effect of DRUG. Bottom row: correlation between FDG metabolic changes (MPH-PL) in the thalamic seeds ( $x$-axes) and in target ROIS ( $y$-axes) shown for each GROUP for a right cerebellum ROI with motor seed, $\mathbf{b}$ right cerebellum ROI with sensory seed, and c NAc ROI formed with pre-motor seed

by blocking norepinephrine transporters (NET) and increasing noradrenergic signaling might have enhanced the connectivity between the thalamus and cerebellum. On the other hand, the NAc primarily receives dopaminergic inputs from the ventral tegmental area, which also projects to thalamic nuclei [48]. The differential effects of MPH's could also reflect that in the NAc both excitatory and inhibitory dopaminergic receptors co-exist (D1-like excitatory versus D2-like inhibitory). MPH could have modulated these receptors differently in $\mathrm{HC}$ and CUD accounting for the decreases in negative connectivity in CUD and the enhanced negative connectivity in $\mathrm{HC}$. Interestingly, $\mathrm{MPH}$ normalized connectivity for CUD, reducing the negative connectivity to the levels in $\mathrm{HC}$ under placebo status, which we interpret to suggest that MPH might have alleviated the dopaminergic deficits in CUD [49-51]. This finding reiterates the pivotal role of NAC and the thalamus in stimulant drugs effects and in addiction [52]. Also, too much or too little dopaminergic signaling might lead to impaired cognitive performance and motivation, which might be mediated in part by thalamo-accumbens connectivity $[53,54]$. Our results are consistent with recent preclinical findings that the inhibition of the paraventricular nucleus by D2Rs influences the NAc affecting cocaine-seeking behavior [21], and our finding of the disruption of thalamo-accumbens connectivity in CUD further support the relevance of this circuit in addiction. Its normalization by MPH also brings forth the possibility of its use as a potential therapeutic strategy to ameliorate connectivity deficits in CUD.
MPH's enhancement of both dopamine and noradrenaline signaling could also underlie the group differences in its metabolic effects. Specifically, if CUD had a predominant disruption of dopaminergic [55] over that of noradrenergic neurotransmission, then this could help explain the similarity in metabolic increases with MPH in CUD and HC in cerebellum, which is predominantly modulated by noradrenergic innervation [56]. A predominant disruption of dopaminergic neurotransmission in CUD [55], could also help explain their lower metabolism in the NAc (for both the $\mathrm{PL}$ and MPH conditions), which is mainly modulated by DA. In addition, an increase in metabolism in HC but not in CUD in the thalamus, might be due to its modulation by both dopaminergic and noradrenergic signals $[57,58]$.

\section{Limitations}

A major limitation in our study is the small sample of participants, which precluded us from assessing the associations between the main imaging findings and the effects of MPH on behavior and cognition. It also limited our ability to assess gender effects, which are relevant to both MPH effects but also CUD. It would have also been desirable to perform the PET and the fMRI measures at the same time rather that in a sequential design but we did not have access to an integrated PET/MRI instrument. Finally in our brain imaging findings in CUD participants we cannot distinguish between consequences of cannabis use and pre-existing characteristics that might have increased the risk for CUD. 


\section{CONCLUSION}

We document significant effects of MPH in long-range thalamic connectivity and these effects differed between HC and CUD. We also showed aberrant negative thalamo-accumbens connectivity and reduced thalamic and NAC metabolism in CUD, which is consistent with disrupted dopaminergic signaling in CUD.

\section{FUNDING AND DISCLOSURE}

This work was accomplished with support from the NIAAA Y1AA3009. The authors declare no competing interests.

\section{ACKNOWLEDGEMENTS}

We would like to thank Karen Torres for her administrative assistance, and Chris Wong for his contribution in data collection. We also thank Joanna Fowler and Anna Konova for their valuable input for the discussions. We would like to thank David Schlyer and Michael Schueller for cyclotron operations; Donald Warner, David Alexoff, and Paul Vaska for PET operations; Colleen Shea, Youwen $\mathrm{Xu}$, Lisa Muench, and Payton King for radiotracer preparation and analysis, Millard Jayne for subject recruitment and Barbara Hubbard and Pauline Carter for patient care.

\section{ADDITIONAL INFORMATION}

Supplementary Information accompanies this paper at (https://doi.org/10.1038/ s41386-018-0287-2).

\section{REFERENCES}

1. Linssen AM, Sambeth A, Vuurman EF, Riedel WJ. Cognitive effects of methylphenidate in healthy volunteers: a review of single dose studies. Int J Neuropsychopharmacol/Off Sci J Coll Int Neuropsychopharmacol. 2014; 17:961-77.

2. Ashok AH, Mizuno Y, Volkow ND, Howes OD. Association of stimulant use with dopaminergic alterations in users of cocaine, amphetamine, or methamphetamine: a systematic review and meta-analysis. JAMA Psychiatry. 2017:74:511-9.

3. Volkow ND, Wang GJ, Tomasi D, Kollins SH, Wigal TL, Newcorn JH, et al. Methylphenidate-elicited dopamine increases in ventral striatum are associated with long-term symptom improvement in adults with attention deficit hyperactivity disorder. J Neurosci: Off J Soc Neurosci. 2012b;32:841-9.

4. Solanto MV. Neuropsychopharmacological mechanisms of stimulant drug action in attention-deficit hyperactivity disorder: a review and integration. Behav brain Res. 1998;94:127-52.

5. Volkow ND, Ding YS, Fowler JS, Wang GJ, Logan J, Gatley JS, et al. Is methylphenidate like cocaine? Studies on their pharmacokinetics and distribution in the human brain. Arch General Psychiatry. 1995;52:456-63.

6. Volkow ND, Fowler JS, Wang G, Ding Y, Gatley SJ. Mechanism of action of methylphenidate: insights from PET imaging studies. J Atten Disord. 2002;6: S31-43.

7. Schabram I, Henkel K, Mohammadkhani Shali S, Dietrich C, Schmaljohann J, Winz $\mathrm{O}$, et al. Acute and sustained effects of methylphenidate on cognition and presynaptic dopamine metabolism: an [18F] FDOPA PET study. J Neurosci: Off J Soc Neurosci. 2014;34:14769-76.

8. Volkow ND, Tomasi D, Wang GJ, Telang F, Fowler JS, Logan J, et al. Predominance of $D 2$ receptors in mediating dopamine's effects in brain metabolism: effects of alcoholism. J Neurosci: Off J Soc Neurosci. 2013;33:4527-35.

9. Volkow ND, Wang GJ, Fowler JS, Logan J, Angrist B, Hitzemann R, et al. Effects of methylphenidate on regional brain glucose metabolism in humans: relationship to dopamine D2 receptors. Am J Psychiatry. 1997a;154:50-55.

10. Wiers CE, Shokri-Kojori E, Wong CT, Abi-Dargham A, Demiral SB, Tomasi D, et al. Cannabis abusers show hypofrontality and blunted brain responses to a stimulant challenge in females but not in males. Neuropsychopharmacol: Off Publ Am Coll Neuropsychopharmacol. 2016a;41:2596-605.

11. Volkow ND, Fowler JS, Wang GJ, Telang F, Logan J, Wong C, et al. Methylphenidate decreased the amount of glucose needed by the brain to perform a cognitive task. PloS One. 2008a;3:e2017.

12. Volkow ND, Tomasi D, Wang GJ, Telang F, Fowler JS, Logan J, et al. Evidence that sleep deprivation downregulates dopamine $D 2 R$ in ventral striatum in the human brain. J Neurosci: Off J Soc Neurosci. 2012a;32:6711-7.

13. Volkow ND, Wang GJ, Telang F, Fowler JS, Logan J, Childress AR, et al. Dopamine increases in striatum do not elicit craving in cocaine abusers unless they are coupled with cocaine cues. Neurolmage. 2008b;39:1266-73.
14. Evers EA, Stiers P, Ramaekers JG. High reward expectancy during methylphenidate depresses the dopaminergic response to gain and loss. Soc Cogn Affect Neurosci. 2017;12:311-8.

15. Konova AB, Moeller SJ, Tomasi D, Volkow ND, Goldstein RZ. Effects of methylphenidate on resting-state functional connectivity of the mesocorticolimbic dopamine pathways in cocaine addiction. JAMA Psychiatry. 2013;70:857-68.

16. Farr OM, Zhang S, Hu S, Matuskey D, Abdelghany O, Malison RT, et al. The effects of methylphenidate on resting-state striatal, thalamic and global functional connectivity in healthy adults. Int J Neuropsychopharmacol/Off Sci J Coll Int Neuropsychopharmacol. 2014;17:1177-91.

17. Ramaekers JG, Evers EA, Theunissen EL, Kuypers KP, Goulas A, Stiers P. Methylphenidate reduces functional connectivity of nucleus accumbens in brain reward circuit. Psychopharmacology. 2013;229:219-26.

18. Volkow ND, Wise RA, Baler R. The dopamine motive system: implications for drug and food addiction. Nat Rev Neurosci. 2017;18:741-52.

19. Schwarz LA, Luo L. Organization of the locus coeruleus-norepinephrine system. Curr Biol: CB. 2015a;25:R1051-6.

20. ODonnell P, Lavin A, Enquist LW, Grace AA, Card JP. Interconnected parallel circuits between rat nucleus accumbens and thalamus revealed by retrograde transynaptic transport of pseudorabies virus. J Neurosci. 1997;17:2143-67.

21. Clark AM, Leroy F, Martyniuk KM, Feng W, McManus E, Bailey MR, et al. Dopamine D2 Receptors in the Paraventricular Thalamus Attenuate Cocaine Locomotor Sensitization. eNeuro. 2017;4:1-17.

22. Matzeu A, Weiss F, Martin-Fardon R. Transient inactivation of the posterior paraventricular nucleus of the thalamus blocks cocaine-seeking behavior. Neurosci Lett. 2015;608:34-39.

23. Navarra RL, Clark BD, Zitnik GA, Waterhouse BD. Methylphenidate and atomoxetine enhance sensory-evoked neuronal activity in the visual thalamus of male rats. Exp Clin Psychopharmacol. 2013;21:363-74.

24. Tomasi D, Volkow ND. Functional connectivity density mapping. Proc Natl Acad Sci USA. 2010;107:9885-90.

25. Konova AB, Moeller SJ, Tomasi D, Goldstein RZ. Effects of chronic and acute stimulants on brain functional connectivity hubs. Brain Res. 2015;1628:147-56.

26. Dasilva M, Grieve KL, Cudeiro J, Rivadulla C. Anandamide activation of CB1 receptors increases spontaneous bursting and oscillatory activity in the thalamus. Neuroscience. 2014;265:72-82.

27. Filbey FM, Schacht JP, Myers US, Chavez RS, Hutchison KE. Marijuana craving in the brain. Proc Natl Acad Sci USA. 2009;106:13016-21.

28. Manza $P$, Tomasi $D$, Volkow ND. Subcortical local functional hyperconnectivity in cannabis dependence. Biol Psychiatry Cogn Neurosci neuroimaging. 2018;3:285-93.

29. Brody AL, Hubert R, Mamoun MS, Enoki R, Garcia LY, Abraham P, et al. Nicotinic acetylcholine receptor availability in cigarette smokers: effect of heavy caffeine or marijuana use. Psychopharmacology. 2016;233:3249-57.

30. Cunningham SI, Tomasi D, Volkow ND. Structural and functional connectivity of the precuneus and thalamus to the default mode network. Human brain Mapp. 2017;38:938-56.

31. Behrens TE, Johansen-Berg $H$, Woolrich MW, Smith SM, Wheeler-Kingshott CA, Boulby PA, et al. Non-invasive mapping of connections between human thalamus and cortex using diffusion imaging. Nat Neurosci. 2003;6:750-7.

32. O'Muircheartaigh J, Keller SS, Barker GJ, Richardson MP. White matter connectivity of the thalamus delineates the functional architecture of competing thalamocortical systems. Cereb cortex. 2015;25:4477-89.

33. Cho ZH, Son YD, Kim HK, Kim NB, Choi EJ, Lee SY, et al. Observation of glucose metabolism in the thalamic nuclei by fusion PET/MRI. J Nucl Med: Off Publ, Soc Nucl Med. 2011;52:401-4.

34. Mergenthaler $P$, Lindauer U, Dienel GA, Meisel A. Sugar for the brain: the role of glucose in physiological and pathological brain function. Trends Neurosci. 2013;36:587-97.

35. Rothman DL, De Feyter HM, de Graaf RA, Mason GF, Behar KL. 13C MRS studies of neuroenergetics and neurotransmitter cycling in humans. NMR Biomed. 2011:24:943-57.

36. Volkow ND, Wang GJ, Telang F, Fowler JS, Alexoff D, Logan J, et al. Decreased dopamine brain reactivity in marijuana abusers is associated with negative emotionality and addiction severity. Proc Natl Acad Sci USA. 2014a;111:E3149-56.

37. Wiers CE, Shumay E, Cabrera E, Shokri-Kojori E, Gladwin TE, Skarda E, et al. Reduced sleep duration mediates decreases in striatal D2/D3 receptor availability in cocaine abusers. Transl Psychiatry. 2016b;6:e752.

38. Friston KJ, Worsley KJ, Frackowiak RS, Mazziotta JC, Evans AC. Assessing the significance of focal activations using their spatial extent. Hum Brain Mapp. 1994:1:210-20

39. RCoreTeam. R: A language and environment for statistical computing. Vienna, Austria: R Foundation for Statistical Computing; 2013.

40. Volkow ND, Wang GJ, Gatley SJ, Fowler JS, Ding YS, Logan J, et al. Temporal relationships between the pharmacokinetics of methylphenidate in the human 
brain and its behavioral and cardiovascular effects. Psychopharmacology. 1996;123:26-33.

41. Zhu Y, Wienecke CF, Nachtrab G, Chen X. A thalamic input to the nucleus accumbens mediates opiate dependence. Nature. 2016;530:219-22.

42. Riano Barros DA, McGinnity CJ, Rosso L, Heckemann RA, Howes OD, Brooks DJ, et al. Test-retest reproducibility of cannabinoid-receptor type 1 availability quantified with the PET ligand [11C] MePPEP. Neurolmage. 2014; 97:151-62.

43. Iversen L. Cannabis and the brain. Brain: a J Neurol. 2003;126:1252-70.

44. Schmahmann JD. The role of the cerebellum in cognition and emotion: personal reflections since 1982 on the dysmetria of thought hypothesis, and its historical evolution from theory to therapy. Neuropsychol Rev. 2010; 20:236-60.

45. Canto CB, Onuki Y, Bruinsma B, van der Werf YD, De Zeeuw Cl. The sleeping cerebellum. Trends Neurosci. 2017;40:309-23.

46. Oh M, Kim JS, Oh JS, Lee CS, Chung SJ. Different subregional metabolism patterns in patients with cerebellar ataxia by $18 \mathrm{~F}$-fluorodeoxyglucose positron emission tomography. PloS One. 2017;12:e0173275.

47. Benarroch EE. The locus ceruleus norepinephrine system: functional organization and potential clinical significance. Neurology. 2009;73:1699-704.

48. Ikemoto S. Dopamine reward circuitry: two projection systems from the ventral midbrain to the nucleus accumbens-olfactory tubercle complex. Brain Res Rev. 2007;56:27-78.

49. Madras BK. Dopamine challenge reveals neuroadaptive changes in marijuana abusers. Proc Natl Acad Sci USA. 2014;111:11915-6.
50. Volkow ND, Wang GJ, Fowler JS, Logan J, Gatley SJ, Hitzemann R, et al. Decreased striatal dopaminergic responsiveness in detoxified cocaine-dependent subjects. Nature. 1997b;386:830-3.

51. Volkow ND, Wang GJ, Telang F, Fowler JS, Alexoff D, Logan J, et al. Decreased dopamine brain reactivity in marijuana abusers is associated with negative emotionality and addiction severity. Proc Natl Acad Sci USA. 2014b;111:E3149-56.

52. Scofield MD, Heinsbroek JA, Gipson CD, Kupchik YM, Spencer S, Smith AC, et al. The nucleus accumbens: mechanisms of addiction across drug classes reflect the importance of glutamate homeostasis. Pharmacol Rev. 2016;68:816-71.

53. Volkow ND, Wang GJ, Newcorn JH, Kollins SH, Wigal TL, Telang F, et al. Motivation deficit in ADHD is associated with dysfunction of the dopamine reward pathway. Mol Psychiatry. 2011;16:1147-54.

54. Cools R, D'Esposito M. Inverted-U-shaped dopamine actions on human working memory and cognitive control. Biol Psychiatry. 2011;69:e113-25.

55. Bloomfield MA, Ashok AH, Volkow ND, Howes OD. The effects of $\Delta^{9}$-tetrahydrocannabinol on the dopamine system. Nature. 2016;539:369-77.

56. Woodward DJ, Moises $\mathrm{HC}$, Waterhouse BD, Yeh $\mathrm{HH}$, Cheun JE. The cerebellar norepinephrine system: inhibition, modulation, and gating. Progress brain Res. 1991;88:331-41.

57. Sanchez-Gonzalez MA, Garcia-Cabezas MA, Rico B, Cavada C. The primate thalamus is a key target for brain dopamine. J Neurosci: Off J Soc Neurosci. 2005;25:6076-83.

58. Schwarz LA, Miyamichi K, Gao XJ, Beier KT, Weissbourd B, DeLoach KE, et al. Viralgenetic tracing of the input-output organization of a central noradrenaline circuit. Nature. 2015b;524:88-92. 